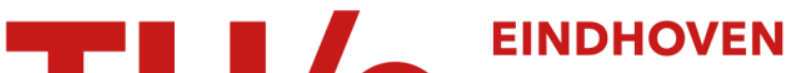 \\ UNIVERSITY OF \\ TECHNOLOGY
}

\section{Structure and conductivity of clusters generated by variable- range hopping percolation}

\section{Citation for published version (APA):}

Huinink, H. P., Bobbert, P. A., Pasveer, W. F., \& Michels, M. A. J. (2006). Structure and conductivity of clusters generated by variable-range hopping percolation. Physical Review B, 73(22), 224204-1/9. [224204]. https://doi.org/10.1103/PhysRevB.73.224204

DOI:

10.1103/PhysRevB.73.224204

Document status and date:

Published: 01/01/2006

\section{Document Version:}

Publisher's PDF, also known as Version of Record (includes final page, issue and volume numbers)

\section{Please check the document version of this publication:}

- A submitted manuscript is the version of the article upon submission and before peer-review. There can be important differences between the submitted version and the official published version of record. People interested in the research are advised to contact the author for the final version of the publication, or visit the $\mathrm{DOI}$ to the publisher's website.

- The final author version and the galley proof are versions of the publication after peer review.

- The final published version features the final layout of the paper including the volume, issue and page numbers.

Link to publication

\section{General rights}

Copyright and moral rights for the publications made accessible in the public portal are retained by the authors and/or other copyright owners and it is a condition of accessing publications that users recognise and abide by the legal requirements associated with these rights.

- Users may download and print one copy of any publication from the public portal for the purpose of private study or research.

- You may not further distribute the material or use it for any profit-making activity or commercial gain

- You may freely distribute the URL identifying the publication in the public portal.

If the publication is distributed under the terms of Article 25fa of the Dutch Copyright Act, indicated by the "Taverne" license above, please follow below link for the End User Agreement:

www.tue.nl/taverne

Take down policy

If you believe that this document breaches copyright please contact us at:

openaccess@tue.nl

providing details and we will investigate your claim. 


\title{
Structure and conductivity of clusters generated by variable-range hopping percolation
}

\author{
H. P. Huinink,* P. A. Bobbert, W. F. Pasveer, and M. A. J. Michels \\ Department of Applied Physics, Technische Universiteit Eindhoven, P.O. Box 513, 5600 MB Eindhoven, The Netherlands
}

(Received 15 February 2006; revised manuscript received 20 April 2006; published 19 June 2006)

\begin{abstract}
An important class of models for variable-range hopping (VRH) transport processes of electrons in highly disordered systems is based on percolation arguments. In these models the so-called critical path analysis (CPA) is combined with percolation arguments based on standard percolation models. Despite the increased computer power in the last decade there have been little attempts to validate the applicability of standard percolation theory on VRH problems. We have performed systematic numerical calculations on the structure and conductivity of VRH percolation clusters in two dimensions. It is shown by analyzing the mass of the clusters and the correlation length that VRH percolation clusters indeed behave as standard percolation clusters. The main difference between VRH percolation and standard percolation seems to be the existence of a temperature dependent effective lattice constant. Conductivity calculations on VRH clusters have been performed that support the central idea behind CPA models. Furthermore, these calculations confirm the existence of critical subnetworks.
\end{abstract}

DOI: 10.1103/PhysRevB.73.224204 PACS number(s): 64.60.Ak, 72.20.Ee, 72.80.Ng, 78.55.Qr

\section{INTRODUCTION}

In not too highly doped disordered semiconductors the states around the Fermi energy have wave functions that are localized at certain sites in the semiconductor. Conduction then takes place by phonon-assisted hopping of electrons or holes from one localized site to another. At low temperatures an electron or hole not necessarily hops between neighboring sites. The hopping distance might be adjusted in order to minimize the energy difference between the two sites involved, called variable-range hopping (VRH). It is well known that the low-temperature conductivity $\Sigma$ in many disordered semiconductors deviates from Arrhenius-type behavior and seems to follow a temperature dependence of the form $\Sigma \propto \exp \left[-\left(T_{0} / T\right)^{\gamma}\right], 0<\gamma<1$. Initially, this temperature dependence was observed in Si-based and Ge-based systems, glasses and III-V compounds. ${ }^{1}$ However, similar behavior has been found in completely different systems such as conjugated polymers, ${ }^{2,3}$ DNA, ${ }^{4}$ and high- $T_{C}$ cuprate oxides. ${ }^{5} \mathrm{An}$ interesting feature is the discrete nature of the parameter $\gamma$, which seems to adopt the values $0.25,0.33$, or 0.50 depending on the dimensionality $d$ of the problem $\left(\gamma \simeq[d+1]^{-1}\right)$.

At the end of the 1960s Mott had derived an equation for the conductivity that could explain the observed temperature dependence and this resulted in an expression for $T_{0}{ }^{6}$ In his derivation he optimized the hopping rate of a single hop from one site to another under the constraint that at least one such hop is possible. A few years later a few more systematic approaches were initiated that were all based on percolation arguments, ${ }^{7,8}$ the so-called critical path analyses (CPA). These studies have in common that they lead to the correct temperature dependency, but differ in their predictions of $T_{0}$. For a detailed overview of the application of percolation theory to conductivity problems we refer to the literature. ${ }^{9-11}$ In CPA approaches the hopping process is mapped onto a random-resistor network. It is argued that the main current paths are on a percolating network, that is built of bonds with the highest conductances and is close to its critical point. Because the distribution of bond conductances is very broad, the conductivity of the percolating network is set equal to the conductance of the bond in the network with the highest resistivity. The conductance of this critical bond can be related to a percolation criterion.

Although percolation arguments are used for many years to describe VRH processes, the number of attempts to compute the properties of the underlying percolation models numerically are limited. Percolation thresholds have been estimated via Monte Carlo calculations. ${ }^{12}$ Most other numerical studies have focussed on calculating $T_{0}$ directly from the conductivity itself. ${ }^{13-15}$ However, the percolation problem that constitutes VRH, to which we will refer as VRH percolation, is more complicated than ordinary site or bond percolation problems. This is clearly illustrated by the key variable $\eta$ of this problem, which is defined as ${ }^{7}$

$$
\eta_{i j} \equiv \alpha R_{i j}+\beta\left\{\left|E_{i}\right|+\left|E_{j}\right|+\left|E_{i}-E_{j}\right|\right\} / 2 .
$$

This variable characterizes a bond between two sites $i$ and $j$ having energies $E_{i}$ and $E_{j}$ and separated by a distance $R_{i j}$. The parameter $\alpha$ is defined as $2 / a$, where $a$ is the localization radius of the wave functions, and $\beta=\left(k_{B} T\right)^{-1}$. Further, we define a variable $\epsilon_{i j} \equiv \beta\left\{\left|E_{i}\right|+\left|E_{j}\right|+\left|E_{i}-E_{j}\right|\right\} / 2$. In VRH percolation clusters and networks are generated by connecting sites by bonds characterized by values of $\eta_{i j}$ below a certain threshold. It follows from the definition of $\eta$ that VRH percolation deviates from standard models for several reasons. First of all, the properties of a bond are strongly related with the properties of the sites via the site energies. Second, the number of bonds that a site $i$ will have with other sites will strongly depend on the value of $\left|E_{i}\right|$. Third, bonds can be made between sites that are not nearest neighbors. Therefore, it is not obvious that VRH percolation belongs to the same universality class as ordinary site and bond percolation, and that the use of standard percolation exponents in VRH problems can be justified.

In this study we want to investigate in more detail the properties of two dimensional networks generated by VRH percolation, in particular their structure and conductive prop- 
erties, and check whether or not VRH percolation models belong to the same universality class as standard percolation models.

\section{THEORY}

\section{A. System}

The system is modelled with the help of a square lattice of $N=L \times 2 L$ sites. The distance between two neighboring sites on the lattice is $l$. In the remainder of this paper length scales will always be scaled by this lattice constant: $\alpha^{\prime} \equiv \alpha l$ and $R^{\prime} \equiv R / l$. To each site $i$ on the lattice a random number $\epsilon_{i}$ is assigned, which is drawn from a uniform distribution $[-\Delta / 2,+\Delta / 2]$. This number equals the dimensionless energy $\beta E_{i}$ at site $i$. Note that the density of states is equal to $n$ $=\beta / l^{2} \Delta$. Further we assume that the Fermi level is located halfway the energy distribution $(\mu=0)$. It has been proven that at sufficiently low temperatures electron hopping in such a system can be thought of as resistor-network problem ${ }^{7}$ in which the conductance of a bond between the sites $i$ and $j$ is given by

$$
g_{i j} \equiv \beta e^{2} g_{0} \exp \left(-\eta_{i j}\right),
$$

where $g_{0}$ is a constant, which depends only weakly on the site energies and the distance between the two sites. According to Eq. (1) the conductance of a bond decreases with an increasing distance $R_{i j}$ between the sites and increasing values of $\left|E_{i}\right|$ and $\left|E_{j}\right|$.

Because the conductance $g_{i j}$ decreases monotonously with $\eta_{i j}$, the latter variable can be used to setup a percolation model for hopping conductivity. With VRH percolation clusters can be built by connecting sites via bonds that obey $\eta_{i j} \leqslant \eta$, where $\eta$ is the threshold value of interest. In the next section we discuss a different approach, which is useful for studying clusters at the percolation threshold.

\section{B. Percolation algorithms}

As already mentioned, percolating clusters can be generated by connecting all sites by bonds given that $\eta_{i j} \leqslant \eta$. We will refer to this approach with VRH-OP (OP stands for ordinary percolation). However, in this study we have mainly grown clusters with a so-called invasion percolation (IP) algorithm, which was originally developed to study multiphase flow problems in porous media. ${ }^{16-18}$ We will use the abbreviation VRH-IP for this approach.

The IP algorithm used in this study is as follows. Cluster growth is initiated at one side of the lattice. At every step in the algorithm one new bond is added, which is chosen as follows. For all bonds that are not part of the cluster, but connect to at least one site of the existing cluster, $\eta_{i j}$ is evaluated. The bond with the lowest value of $\eta_{i j}$ is invaded (added to the cluster). When this bond is in contact with a site that does not participate in the cluster, this site is also added to the cluster. It has to be stressed that our algorithm does not contain so-called trapping rules, which are commonly used in fluid flow problems. ${ }^{20}$ Different criteria can be used to terminate the growth process: (1) $\eta_{i j}>\eta$ or (2) the cluster just spans the complete lattice (called breakthrough).
Whereas in an OP model many different clusters are generated, in an IP model only a single cluster is formed, which is by definition the spanning cluster.

At the time that IP algorithms were introduced, it was believed that a nontrapping IP cluster and a spanning OP cluster have a similar structure. ${ }^{18-20}$ Recent simulations have indeed shown that in the absence of trapping the scaling behavior of spanning IP clusters (mass, backbone, and optimal path) does not differ from OP. ${ }^{21,22}$ The similarity between spanning VRH-IP and VRH-OP clusters can be understood as follows. Consider a very large lattice of blue sites. Every site is connected by blue colored bonds to all other sites in the lattice. A bond $i j$ is labeled with a number $\eta_{i j}$, see Eq. (1). In VRH-OP we color all bonds and connecting sites red with $\eta_{i j} \leqslant \eta$ ( $\eta$ is larger or equal to the percolation threshold $\eta_{c}$ ). As a result we end up with one spanning cluster and a number of isolated clusters of red bonds and sites. In VRH-IP we also color the bonds (and sites) red with $\eta_{i j}$ $\leqslant \eta$. However, now we start at a specific side of the system. At a certain stage of the painting process, we only color a bond (and site) red, given that at least one of two sites that are connected by this bond has been painted red in previous steps of the process. As a consequence, we generate only one cluster that is in contact with both sides of the system. The final red cluster is identical to the spanning cluster, generated with VRH-OP. The end result only differs from VRH-OP by the fact that no isolated red clusters are generated.

The main advantage of an IP algorithm is that it is relatively easy to identify the percolation threshold and the percolating cluster by using the breakthrough criterion. Therefore, IP models are useful for studying scaling properties of percolating clusters. Finally, it has to be remarked that this study is about de conduction to which the isolated clusters do not contribute.

\section{Properties}

The properties of the percolating cluster are calculated in the central $L \times L$ part of the lattice in order to minimize the influence of the boundaries. The number of sites $M_{s}$ and bonds $M_{b}$ participating in the cluster is calculated. The conductivity $\sigma$ of the network is found by solving Kirchhoff's equations at every lattice site $i$ with a conjugate gradient method

$$
\sum_{j=1}^{N} g_{i j}\left(V_{i}-V_{j}\right)=0,
$$

where $V_{i}$ and $V_{j}$ are the potentials at the site $i$ and $j$, respectively. Note that $g_{i j}=0$ when the sites $i$ and/or $j$ are not part of the percolating network. The potentials at sites outside the central region are set to either $V_{\min }$ or $V_{\max }\left(\Delta V \equiv V_{\max }\right.$ $\left.-V_{\text {min }}\right)$. The conductivity can be calculated from the total current $I$ with the following expression:

$$
\Sigma \equiv \frac{J}{E}=\frac{I}{|\Delta V|}\left(\frac{L+1}{L}\right),
$$

where $|J|=I / L l$ and $E=\Delta V /(L+1) l$ are the current flux and electrical field, respectively. From the current distribution we 


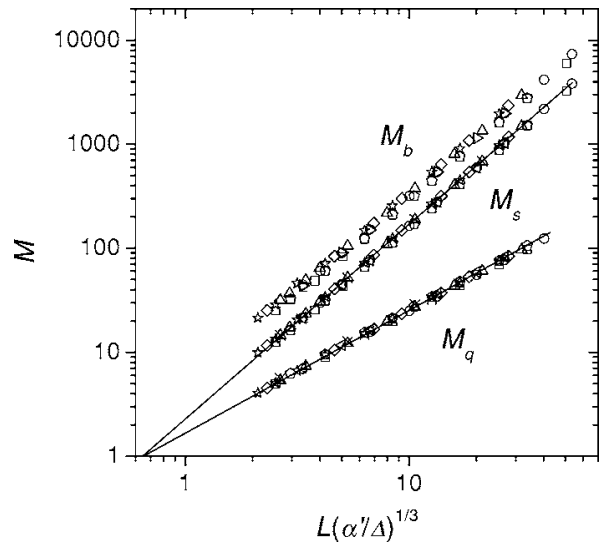

FIG. 1. The number of sites $M_{s}$, the number of bonds $M_{b}$, and the number of sites in the main current path in a cluster $M_{q}$ for different values of $\alpha^{\prime}$ and $\Delta$ as calculated with VRH-IP. Results are shown for $\alpha^{\prime}=3, \Delta=40(\square), 80(\bigcirc), 160(\triangle), 240(\diamond)$, and 320 $(\succsim)$, and $\alpha^{\prime}=6, \Delta=80(\square), 160(\triangleleft)$, and $320(\times)$. All data points are averages over 1000 realizations.

identify the current path with the highest current output at the downstream side of the central box and calculate the number of sites in this path $M_{q}$. This path is constructed by taking the site that feeds the last one with the highest current flow. This procedure is repeated until the upstream side of the lattice is reached. In the rest of this study we will refer to this path as the main current path.

The properties $M_{s}, M_{b}, M_{q}$, and $\ln \Sigma$ are calculated as averages over various realizations. Also the standard deviations of these properties are calculated.

\section{NUMERICAL RESULTS}

\section{A. Structure}

\section{Mass of percolating cluster}

A percolation cluster or a subset of this cluster can be characterized by its mass, i.e., the number of sites or bonds in the cluster. We have calculated the number of sites $M_{s}$, the number of bonds $M_{b}$, and the number of sites in the main current path in a cluster $M_{q}$ for different values of $\alpha^{\prime}$ and $\Delta$ with VRH-IP. In Fig. 1 we have plotted $M_{s}$ and $M_{q}$ as a function of the system size $L$ divided by a scale $\left(\Delta / \alpha^{\prime}\right)^{1 / 3}$. We have chosen to rescale $L$ in this way because the analysis of Mott already made clear that a length scale with such a behavior is involved in VRH processes. ${ }^{6}$

From the fact that all data points collapse on master curves, it can be concluded that there exists a characteristic length scale $r=\rho\left(\Delta / \alpha^{\prime}\right)^{1 / 3}$ ( $\rho$ is a constant). By extrapolating the curves to $M_{s}=M_{q}=1$ we can obtain an estimate for this length scale: $\rho \simeq 0.65 \pm 0.05$. The length scale $r$ proves to be close to the average bond length $\left\langle R^{\prime}\right\rangle$. In a system where $\Delta$ $=160$ and $\alpha=3$ the average bond length $\left\langle R^{\prime}\right\rangle$ decreases from 0.66 to $0.63\left(\Delta / \alpha^{\prime}\right)^{1 / 3}$ when the system size $L$ increases from 10 to 120 . From this we conclude that changes in the roughness of the energy landscape $\Delta$ (in fact the temperature) or the inverse decay length of the wave functions $\alpha^{\prime}$ mainly modify the distance between two neighboring sites in a cluster; $r$ can be thought of as an effective lattice constant. The scaling of $r$ with $\left(\Delta / \alpha^{\prime}\right)^{1 / 3}$ can be understood as follows. When a system has a typical effective lattice constant $r$, then it follows from Eq. (1) that a typical expression for $\eta$ behaves as

$$
\eta=\alpha^{\prime} r+\epsilon \sim \alpha^{\prime} r+\frac{\Delta}{r^{2}} .
$$

Note that within an area $r^{2}$ the typical spacing in the energy levels $\epsilon \sim \Delta / r^{2}$. Because we build the clusters from bonds having the lowest values of $\eta$, it can be derived by minimization of Eq. (5) with respect to $r$ that

$$
r \sim\left(\frac{\Delta}{\alpha^{\prime}}\right)^{1 / 3} .
$$

In the derivation of this equation we have followed a similar reasoning as Mott used in his derivation of the optimal hopping distance. ${ }^{6}$

The observed scaling behavior of the effective lattice constant will only hold as long as $r$ exceeds the distance between two neighbor sites on the underlying lattice; the following criterion has to be satisfied

$$
\left(\frac{\Delta}{\alpha^{\prime}}\right)^{1 / 3}>\rho^{-1} .
$$

Only when this criterion is met, one can speak of VRH percolation. When this criterion is not met, sites in the cluster are by definition nearest neighbors and our problem reduces to a type of correlated site-bond percolation problem that has been discussed in the literature. ${ }^{23}$ In the case that $\alpha^{\prime}=3$, $\Delta>12$ is required to have $\mathrm{VRH}$ percolation.

The fractal nature of the percolating cluster is revealed by the power-law nature of the curves in Fig. 1. By fitting we have obtained the fractal dimensions $D_{s}=1.85 \pm 0.01$ and $D_{q}=1.15 \pm 0.01$ for the total number of sites, $M_{s}$, and the number of sites in the current path, $M_{q}$, respectively. The value of $D_{s}$ is close to the value reported for ordinary percolation models, $D_{s}=91 / 48 .{ }^{24}$ The value of $D_{q}$ is close to the value associated with the minimal path on a cluster generated by an ordinary percolation process, $D_{\min }$, which equals 1.13 or 1.17 at or above the percolation threshold, respectively. ${ }^{24}$ This shows that the path is still important in VRH conduction and its length is minimized as much as possible. The values of both fractal dimensions suggest that VRH percolation belongs to the same universality class as ordinary site or bond percolation.

\section{Coordination number}

Another property that contains information on the cluster structure is the average number of bonds $M_{b}$ per site $B$ $\equiv M_{b} / M_{s}$ ( $B$ goes to a fixed value $B_{c}$ for $\left.L \rightarrow \infty\right)$. In Fig. 2 we have plotted the values of $B$ that were obtained for the largest systems $(L=120)$ as function of $\Delta / \alpha^{\prime}\left(B \approx B_{c}\right)$. The critical coordination varies with $\Delta / \alpha^{\prime}$ and seems to converge to a constant value $B_{c, \infty}$ for $\Delta / \alpha^{\prime} \rightarrow \infty$. We were able to fit the data very well with the following expression: 


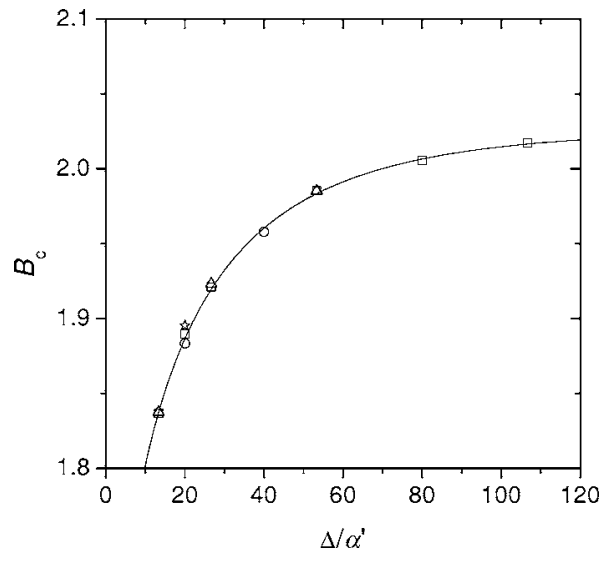

FIG. 2. The average number of bonds per site, $B_{c}=M_{b} / M_{s}$, for various large systems $(L=120$ or 160$)$ with $\alpha^{\prime}=3(\square), 4(\bigcirc), 6$ $(\triangle)$, and $8(\succsim)$. The solid line represents a fit through the data points with an expression of the type of Eq. (8).

$$
B_{c}=B_{c, \infty}-\Delta B \exp \left\{-\left[\left(\Delta / \alpha^{\prime}\right)^{1 / 3} / \lambda\right]^{2}\right\},
$$

where $\Delta B$ is a constant. The solid line in Fig. 2 represents a fit with $B_{c, \infty}=2.03, \Delta B=0.50$, and $\lambda=2.41$.

The fact that $B_{c}$ is a function of $\left(\Delta / \alpha^{\prime}\right)^{2 / 3}$ can be understood as follows. As we have seen in the previous section, the average bond length $r$ in the percolating cluster is proportional to $\left(\Delta / \alpha^{\prime}\right)^{1 / 3}$. Therefore, $\left(\Delta / \alpha^{\prime}\right)^{2 / 3}$ is proportional to the area around a site, needed to have enough other sites to connect with in order to generate a percolating cluster. When the structure of our percolating cluster, reflected in $B_{c}$, would have been invariant for the choice of $\alpha^{\prime}$ and $\Delta$, the behavior captured by Eq. (8) would not have been observed. However, this equation suggests that there exists another intrinsic length scale $\rho \lambda$ besides $r$. From the fit it follows that there is a subtle change in the local structure of the cluster at $r$ $\approx \rho \lambda$. Below this length scale the number of bonds that have to participate in an infinite cluster decreases with decreasing value of $r$.

Surprisingly, the values obtained for $B_{c}$ for high values of $\Delta / \alpha^{\prime}$ are close to the ones reported for ordinary percolation, $B_{c} \simeq d /(d-1)$ with $d$ as the euclidean dimension. ${ }^{9,25}$ At low values of $\Delta / \alpha^{\prime}$ the value of $B_{c}$ is lower, which could indicate that bonds are stronger correlated on a local scale (the percolation threshold is generally lower in correlated percolation $\left.^{23,26}\right)$.

\section{Percolation variable}

In standard site OP models the state of the system is determined by the probability $p$ that a site participates in a cluster (one could also refer to $p$ as the concentration of sites). In VRH percolation the state of the system can be characterized by $\eta$, see Eq. (1). All bonds (and sites connected with these bonds) that have a value of $\eta$ below a certain threshold value participate in clusters. In this section we want to investigate the relation between the threshold value of $\eta$ and $p$.

In order to clarify this relation we have calculated the number of sites $N_{s}$ that are connected by bonds that obey

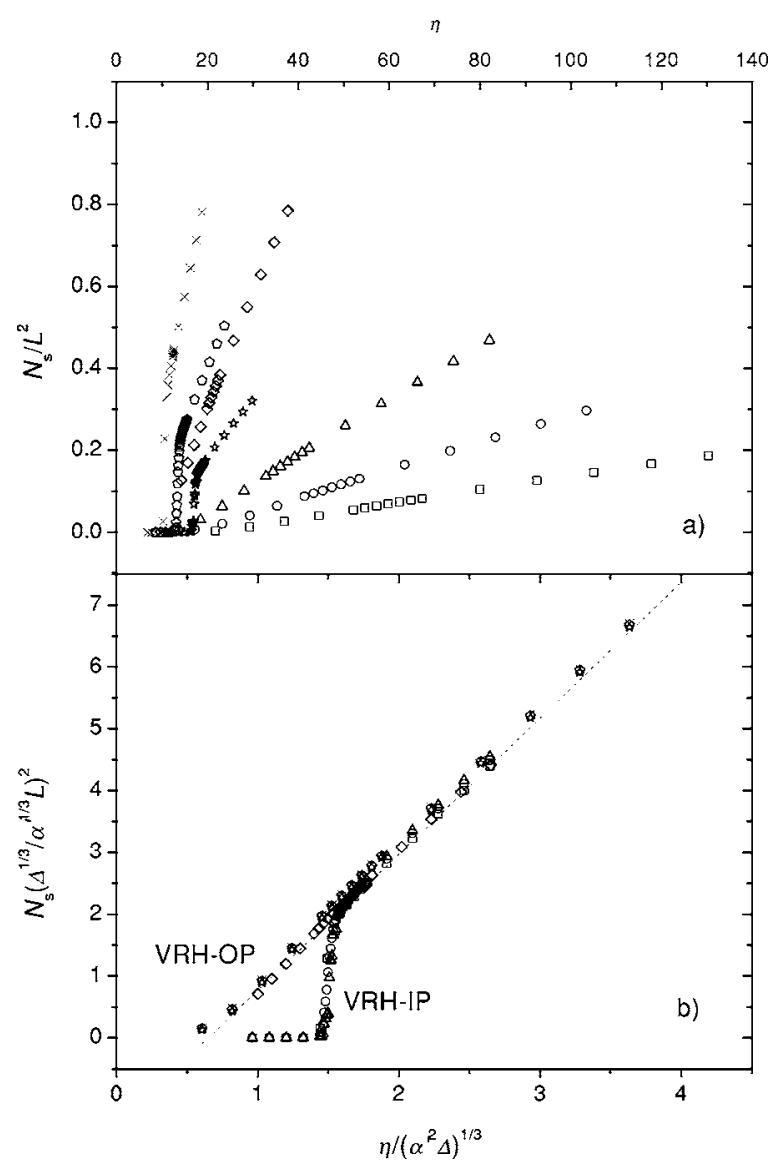

FIG. 3. The "concentration" of sites $p=N_{s} / L^{2}$ participating in clusters built from bonds with $\alpha^{\prime} R^{\prime}+\epsilon \leqslant \eta$ (a) and the scaled concentration of sites $N_{s} /\left[L\left(\alpha^{\prime} / \Delta\right)^{1 / 3}\right]^{2}$ vs the scaled percolation variable, $\eta / \alpha^{\prime 2 / 3} \Delta^{1 / 3}$ (b). The data points for $\alpha^{\prime}=3$ were calculated with VRH-IP: $\Delta=40(\square), 80(\bigcirc)$ and $160(\triangle)$. With VRH-OP calculations were done with $\alpha^{\prime}=6: \Delta=80(\diamond), 320(\diamond), 640(\square)$, and $1280(\times)$. The dashed line represents a fit through the data with Eq. (13).

$\alpha^{\prime} R^{\prime}+\epsilon \leqslant \eta$. With VRH-OP and VRH-IP algorithms calculations were performed on systems with $\alpha^{\prime}=3$ and $\alpha^{\prime}=6$. In Fig. 3(a) we have plotted $p=N_{s} / L^{2}$ as a function of $\eta$. By scaling $L$ with $(\Delta / \alpha)^{1 / 3}$ and $\eta$ with $\left(\alpha^{\prime 2} \Delta\right)^{1 / 3}$ all data points collapse on mastercurves for VRH-IP and VRH-OP, see Fig. 3(b). The dotted line in Fig. 3(b) represents a fit of the VRH-OP data (see below).

Figure 3 shows that the concentration of sites $p$ that participate in VRH-OP clusters is a unique and rather linear function of $\eta$. While at high values of $\eta$ the VRH-IP curves tend to coincide with the VRH-OP, large deviations are observed at lower values. This can be understood as follows. In VRH-OP clusters of sites are built simultaneously and can be located everywhere in the system. In VRH-IP one cluster is generated, which only above a certain critical value $\eta_{c}$ contains a significant amount of sites and spans the system.

In order to explain the scaling behavior, illustrated by Fig. 3 , we adopt the following renormalization procedure. As discussed in the previous sections, the smallest scale of the structure of a VRH cluster is governed by the length scale $r=\rho\left(\Delta / \alpha^{\prime}\right)^{1 / 3}$. We now replace the original lattice by a new 


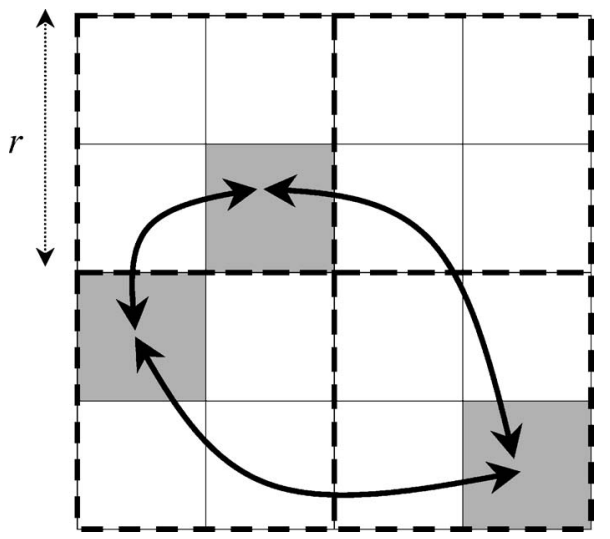

FIG. 4. Schematical picture of the renormalization procedure. The original lattice sites are represented by the small squares. Sites that participate in the percolation cluster are colored gray. In this particular example four sites are grouped together in cells with a squared shape (dashed lines) and a typical spacing $r=2$. The percolation cluster can be thought of as a cluster of cells that contain a gray square.

lattice with a dimensionless spacing $r$ and cells with a certain geometry. In the example shown in Fig. 4 cells with a square shape are used. Within a cell $m=K r^{2}$ sites of the original lattice are grouped together and viewed as an effective site ( $K$ is geometrical constant of order unity). The probability that such an effective site participates in a cluster is $\phi$ $\simeq K N_{s}(r / L)^{2}$. Its energy $\widetilde{E}$ is set equal to the energy of the site within the corresponding square in the original system closest to the Fermi energy, $\widetilde{E}=\{E\}_{\min }$. In fact we assume that clusters are built from sites with energies closest to the Fermi level. The renormalized distribution of site energies obeys the following expression:

$$
P(\beta \widetilde{E})=\frac{K r^{2}}{\Delta} \exp \left(-2 \beta|\widetilde{E}| K r^{2} / \Delta\right) .
$$

For a derivation of this expression we refer to the appendix. By assuming that a percolation cluster is built of cells with energies closest to the Fermi level and using Eq. (9), we can obtain a relation between $\phi$ and the typical energy scale $\epsilon$ of the problem

$$
\phi=\int_{-\epsilon / 2}^{\epsilon / 2} P(u) d u=1-\exp \left(-K r^{2} \epsilon / \Delta\right) .
$$

When $K r^{2} \epsilon / \Delta<1$ the above expression can be simplified to

$$
\phi \simeq K r^{2} \epsilon / \Delta .
$$

Two systems, characterized by different values of $\Delta$ and $\alpha^{\prime}$, are similar when they have the same value of $\phi$ ( $\phi$ can be interpreted as a state variable). By combining Eqs. (1) and (11), an expression for the variable $\eta$ can be derived

$$
\eta \simeq \alpha^{\prime} r+\frac{K \phi \Delta}{r^{2}}
$$

This equation can be rewritten as,

$$
\frac{N_{s}\left(\Delta / \alpha^{\prime}\right)^{2 / 3}}{L^{2}} \simeq K^{-1}\left(\frac{\eta}{\alpha^{\prime 2 / 3} \Delta^{1 / 3}}-\rho\right) .
$$

Despite the number of approximations involved, this equation describes the data surprisingly well, see Fig. 3. The point where $N_{s}=0$ indeed coincides with $\eta / \alpha^{\prime 2 / 3} \Delta^{1 / 3}=\rho$. By fitting we have obtained $K \simeq 0.45$ (dotted line).

From Fig. 3 we can also obtain a first estimation for the percolation threshold, $\eta_{c} \simeq 1.5 \alpha^{\prime 2 / 3} \Delta^{1 / 3}$, which is in good agreement with previous predictions of Seager and Pike, ${ }^{14}$ $C_{2} \equiv \sqrt[3]{4} \eta_{c} /\left(\alpha^{\prime 2} \Delta\right)^{1 / 3}=2.42$, and Skal and Shlovskii, ${ }^{12} \tilde{\lambda}$ $\equiv 4 \eta_{c}^{3} / \alpha^{\prime 2} \Delta=13.8$. From $\eta_{c}$ we estimate $\phi_{c} \simeq 0.68$ agreeing with critical area fractions reported in the literature. ${ }^{13,27}$

\section{Percolation threshold}

Although we have already estimated the percolation threshold in the previous section, we have determined percolation thresholds more accurately with VRH-IP as the value of $\eta=\eta_{c l}$ at which a system spanning network is formed $\left(\eta_{c l}\right.$ is the percolation threshold for one particular realization of a system with size $L$ ). For given values of $\alpha, \Delta$, and $L$ we have obtained distributions of threshold values. In this section we analyse the average value $\left\langle\eta_{c l}\right\rangle$ and the standard deviation $\sigma_{\eta}$ of these distributions. Since we have shown in the previous section that $\eta$ varies linear with the "concentration" of sites in the system, we expect these parameters to scale with the system size $L$ as follows: ${ }^{24,25}$

$$
\left\langle\eta_{c l}\right\rangle-\eta_{c} \propto L^{-1 / \nu}
$$

and

$$
\sigma_{\eta}=\sqrt{\left\langle\eta_{c l}^{2}\right\rangle-\left\langle\eta_{c l}\right\rangle^{2}} \propto L^{-1 / \nu},
$$

where $\nu$ is the correlation length exponent $[\nu=4 / 3$ in two dimensions $^{24}$ (2D)].

The average percolation thresholds are shown in Fig. 5. In Fig. 5(a) we have plotted $\left\langle\eta_{c l}\right\rangle /\left(\alpha^{\prime 2} \Delta\right)^{1 / 3}$ as a function of the scaled system size $L\left(\alpha^{\prime} / \Delta\right)^{1 / 3}$. Although the curves confirm our previous observation that $\eta_{c} \simeq 1.5 \alpha^{\prime 2 / 3} \Delta^{1 / 3}$, a significant spread is observed. A better result is obtained by scaling $\left\langle\eta_{c l}\right\rangle$ also with $B_{c}^{1 / 3}$, Fig. 5(b), which can be understood as follows. Already in the seventies it was proposed that the correct percolation threshold should depend on the average coordination number at percolation, $B_{c}$ (Ref. 14) and we have shown that $B_{c}$ slightly varies with the ratio of $\alpha^{\prime} / \Delta$, Fig. 2. From the data we have estimated the percolation threshold of an infinite system to be $\eta_{c} /\left(\alpha^{\prime 2} B_{c} \Delta\right)^{1 / 3}=1.205 \pm 0.005$. We have fitted the average percolation thresholds with an expression of the type $L^{-1 / \omega}+$ const and have obtained $\omega=0.83$ [the fit is represented by the solid line in Fig. 5(b)]. The fact that $\omega \neq 4 / 3$ does not mean that the value of the correlation length exponent $\nu$ has changed. According to Eq. (15) changes in the value of the correlation length exponent $\nu$ should also show up in the size dependency of $\sigma_{\eta}$. Therefore, we have also plotted $\sigma_{\eta} /\left(\alpha^{\prime 2} B_{c} \Delta\right)^{1 / 3}$ as a function of $L^{-1 / \nu}\left(\alpha^{\prime} / \Delta\right)^{-1 / 3 \nu}$ with $\nu=4 / 3$, see Fig. 6 . The data converge to a straight line indicating that expression (15) holds and the correlation length exponent is not modified by VRH $(\nu$ $=4 / 3$ ). Therefore, we conclude that the expression (14) does 


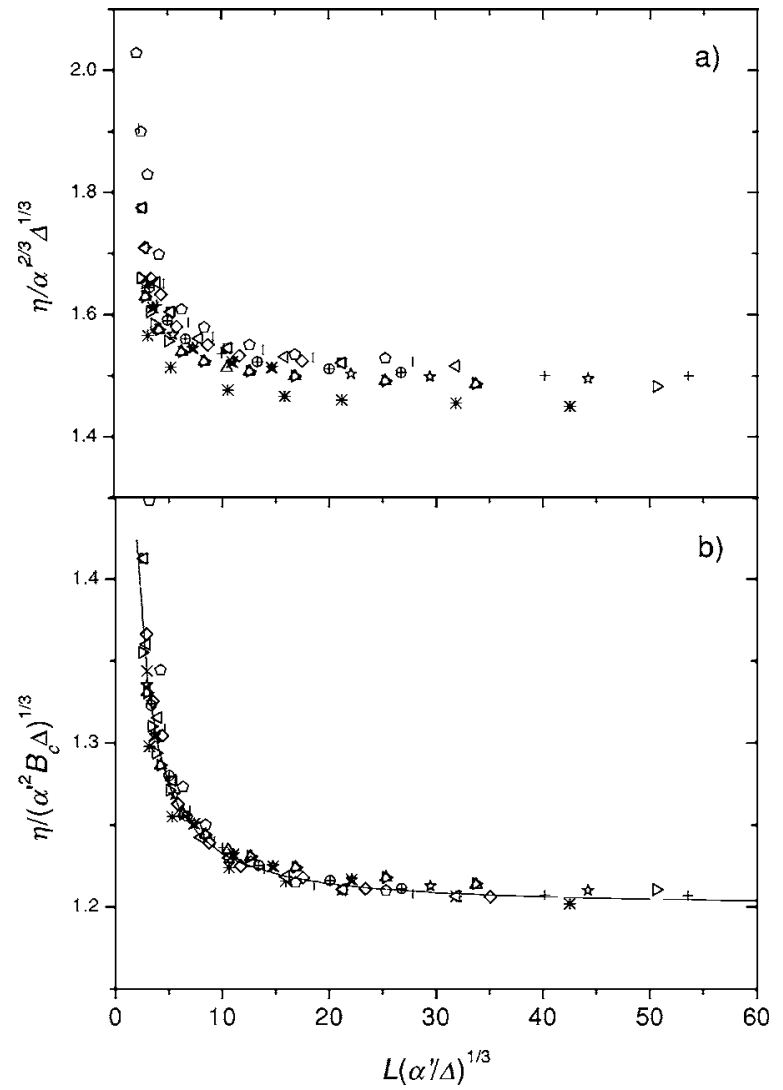

FIG. 5. Scaled percolation thresholds, (a) $\left\langle\eta_{c l}\right\rangle /\left(\alpha^{\prime 2} \Delta\right)^{1 / 3}$ or (b) $\eta_{c l} /\left(\alpha^{\prime 2} B_{c} \Delta\right)^{1 / 3}$, as a function of the scaled system size, $L\left(\alpha^{\prime} / \Delta\right)^{1 / 3}$. Every individual data point is an average of 1000 realizations. Calculations have been performed for $\alpha^{\prime}=3, \Delta=20(*), 40(\triangleright), 80$ $(+), 160(\triangleleft), 240(\mid)$, and $320(\square), \alpha^{\prime}=4, \Delta=80(\succsim)$ and $160(\diamond)$, $\alpha^{\prime}=6, \Delta=80(\triangle), 160(\bigcirc)$, and $320(\square)$, and $\alpha^{\prime}=8, \Delta=160(\times)$. The solid line represents a fit through the data with a function of type $L^{-1 / \omega}+$ const with $\omega=0.83$.

not hold, which is in agreement with observations that were reported in studies on ordinary 2D site percolation. ${ }^{28-30}$

\section{B. Conductivity}

\section{At the percolation threshold}

In this section we investigate in more detail the conductivity of critical networks, e.g., clusters just at percolation. Given that on such a critical cluster there is only one path that significantly contributes to the conductivity of the cluster, the resistance of the cluster is the sum of the resistances of the bonds forming this path. Therefore, the conductivity of the cluster can be written as follows:

$$
\begin{aligned}
\Sigma & =L^{2-d}\left[\sum_{k=1}^{N}\left(\beta e^{2} g_{0}\right)^{-1} \exp \eta_{k}\right]^{-1} \\
& \equiv L^{2-d} \beta e^{2} g_{0} \exp \left(-\eta_{c l}\right) N_{e f f}^{-1},
\end{aligned}
$$

where $N_{e f f} \equiv 1+\sum_{k=2}^{N} \exp \left(\eta_{k}-\eta_{c l}\right)$ can be thought of as the effective number of bonds that determine the conductivity of the cluster (the bonds with the lowest conductances). We

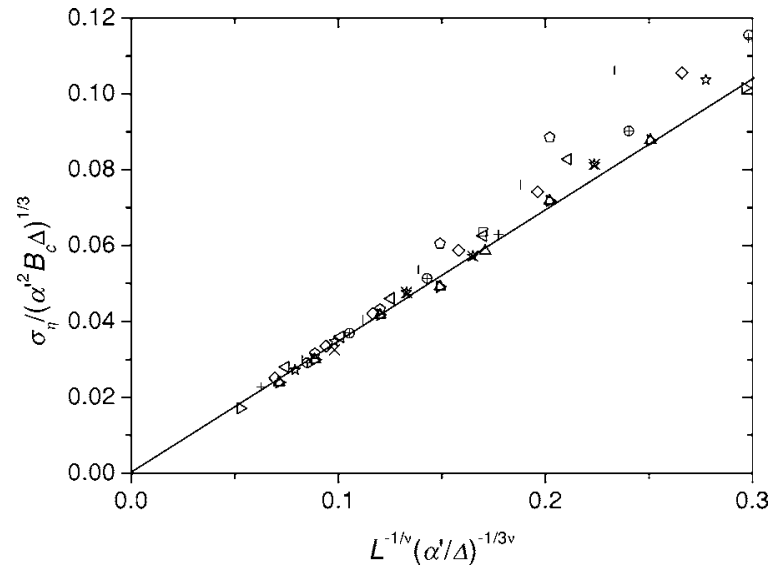

FIG. 6. The scaled standard deviation of the percolation threshold, $\sigma_{\eta} /\left(\alpha^{\prime 2} B_{c} \Delta\right)^{1 / 3}$, as a function of $L^{-1 / \nu}\left(\alpha^{\prime} / \Delta\right)^{-1 / 3 \nu}$ with $\nu$ $=4 / 3$. For the labels of the data points we refer to Fig. 5 . The solid line represents the case that $\sigma_{\eta} \propto L^{-1 / \nu}$.

have chosen the bond $k=1$ as the bond with the lowest conductance, $g=\beta e^{2} g_{0} \exp \left(-\eta_{c l}\right)$. It follows from Eq. (16) that $N_{e f f}=1$ and $-\ln \left(\Sigma / \beta e^{2} g_{0}\right)=\eta_{c l}$ for broad distributions of bond conductances.

Due to the prefactor $L^{2-d}$ of Eq. (16) a 2D system is an interesting case. In two dimensions, $d=2$, the conductivity does not depend explicitly on the system size. When a size dependency of the conductivity is found in such a system, it can be attributed without any doubt to the fact that more bonds than one determine the resistance of the cluster $\left(N_{\text {eff }}>1\right)$.

In Fig. 7 the cumulative distributions of $\eta_{c l}$ (dotted lines) and $-\ln \left(\Sigma / \beta e^{2} g_{0}\right)$ (solid lines) are plotted for systems with $\alpha^{\prime}=6$ and $\Delta=160$ for different system sizes. In small systems $\left(L=10,15\right.$, and 20) the distributions of $\eta_{c l}$ and $-\ln \left(\Sigma / \beta e^{2} g_{0}\right)$ coincide, indicating that the conductivity of the cluster equals the conductance of the critical bond, $\Sigma$ $=\beta e^{2} g_{0} \exp \left(-\eta_{c l}\right)$. For $L=10$ we have calculated $\left\langle N_{e f f}\right\rangle \simeq 1$. Significant deviations between the curves of both distribu-

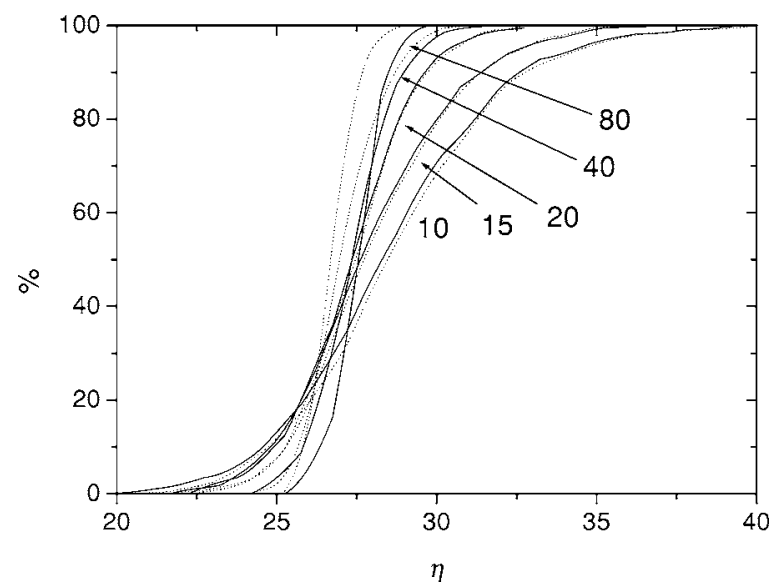

FIG. 7. The cumulative distributions of percolation thresholds, $\eta_{c l}$ (dotted lines), and the logarithm of the cluster conductivities, $-\ln \left(\Sigma / \beta e^{2} g_{0}\right)$ (solid lines), for different system sizes, $L=10,15,20$, 40 , and 80 . 


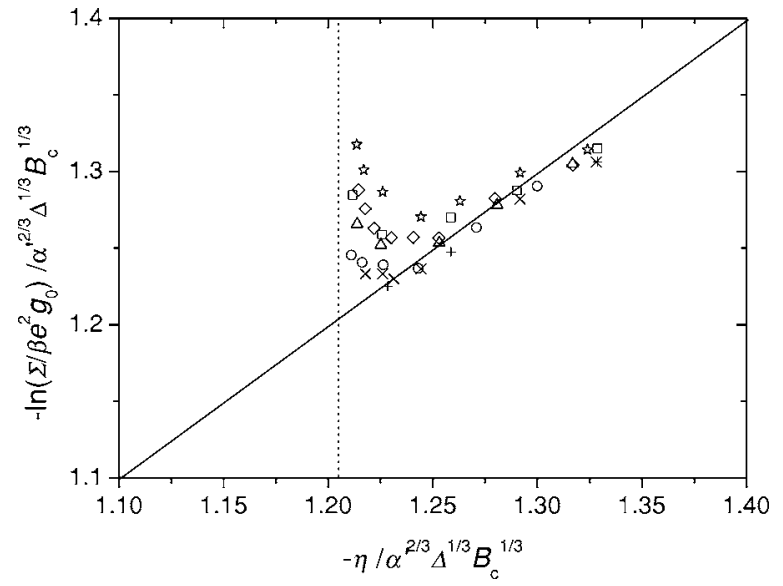

FIG. 8. The average of $-\ln \left(\Sigma / \beta e^{2} g_{0}\right)$ as a function of the average of $\eta_{c l}$. Both variables have been scaled on $\left(\alpha^{\prime 2} B_{c} \Delta\right)^{1 / 3}$. The different symbols refer to different systems: $\Delta=80, \alpha^{\prime}=4$ (它) and $6(\diamond), \Delta=160, \alpha^{\prime}=3(\square), 4(\triangle), 6(\bigcirc)$, and $8(\times), \Delta=320, \alpha^{\prime}$ $=6(+)$. The dotted line represents the percolation threshold and the solid line refers to the case that $\left\langle\ln \left(\Sigma / \beta e^{2} g_{0}\right)\right\rangle=-\left\langle\eta_{c l}\right\rangle$.

tions are observed for the larger systems $(L=40$ and 80$)$. In this case $\Sigma<\beta e^{2} g_{0} \exp \left(-\eta_{c l}\right)$, which means that the conductivity of the clusters is determined by more than one bond, $N_{e f f}>1$. For $L=80$ we obtained $\left\langle N_{e f f}\right\rangle \simeq 3$.

To analyze the relation between the distributions of cluster conductivities $-\ln \left(\Sigma / \beta e^{2} g_{0}\right)$ and percolation thresholds $\eta_{c l}$ for a variety systems, it is easier to characterize these distributions by their average values and standard deviations. We will use $\sigma_{\Sigma}$ to refer to the standard deviation of $-\ln \left(\Sigma / \beta e^{2} g_{0}\right)$, which is given by

$$
\sigma_{\Sigma}=\sqrt{\left\langle(\ln \Sigma)^{2}\right\rangle-\langle\ln \Sigma\rangle^{2}}
$$

In Fig. 8 we have plotted $-\left\langle\ln \left(\Sigma / \beta e^{2} g_{0}\right)\right\rangle$ as a function of $\left\langle\eta_{c l}\right\rangle$. The solid line represents the case that the cluster conductivity is completely determined by the conductance of the critical bond, $N_{e f f}=1$. In small systems $-\left\langle\ln \left(\Sigma / \beta e^{2} g_{0}\right)\right\rangle$ $\simeq\left\langle\eta_{c l}\right\rangle$, indicating that the conductivity of the clusters is indeed determined by a single bond, $N_{e f f}=1$. With increasing system size the influence of other bonds become more pronounced. Close to the percolation threshold for the infinite cluster $\eta_{c}$, the vertical dotted line, $\left.-\left\langle\ln \left(\Sigma / \beta e^{2} g_{0}\right)\right\rangle\right\rangle\left\langle\eta_{c l}\right\rangle$, which is a consequence of the fact that the conductivity of the clusters is no longer determined by one critical bond solely. It seems reasonable to assume that $N_{\text {eff }}$ diverges at $\eta_{c}$.

In Fig. $9 \sigma_{\Sigma}$ is plotted as a function of $\sigma_{\eta}$. Again the solid line represents the case that $N_{\text {eff }}=1$. From this graph we conclude that the fluctuations in $-\ln \left(\Sigma / \beta e^{2} g_{0}\right)$ are less sensitive to the system size than the average value, which indicates that the fluctuations in $\ln N_{\text {eff }}$ play a minor role.

Our results confirm the existence of the so-called critical sub-network. ${ }^{10,11,31}$ In many CPA approaches it is stated that the conductivity is determined by the bonds with a somewhat higher resistance than the resistance of the critical bond. Therefore, it is assumed that the percolation network is close to criticality but has a finite correlation length. Especially Fig. 8 nicely demonstrates this idea. A just percolating net-

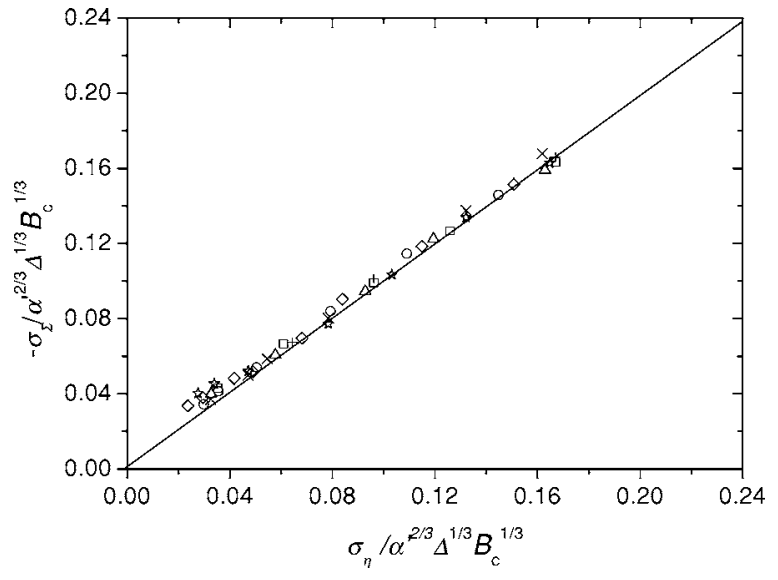

FIG. 9. The standard deviation of $-\ln \left(\Sigma / \beta e^{2} g_{0}\right)$ as a function of the standard deviation of $\eta_{c l}$. Both variables have been scaled on $\left(\alpha^{\prime 2} B_{c} \Delta\right)^{1 / 3}$. The different symbols refer to different values of $\alpha^{\prime}$ and $\Delta$ (see the caption of Fig. 8).

work has a minimal resistance at a finite system size, which is a measure for the length scale of the critical subnetwork.

However, our findings disagree with CPA theories about the origin of the critical subnetwork in 2D. In CPA approaches the critical subnetwork is identified as follows. ${ }^{10,11,31}$ The length scale $L$ in Eq. (8) is related to $\eta_{c l}$ and $N_{e f f}$ is assumed to be equal to one (the central assumption of CPA). Then $\Sigma$ is optimized with respect to $\eta_{c l}$. However, this procedure fails in 2D, because in $2 \mathrm{D}$ the prefactor of Eq. (8) $L^{2-d}=1$. As mentioned in the discussion of Eq. (8), a system size dependency of the conductivity in $2 \mathrm{D}$ makes clear that the cluster resistance is determined by multiple bonds $\left(N_{\text {eff }}>1\right)$. Our results therefore indicate that in 2D a critical subnetwork is formed, because close to the percolation threshold the cluster resistance increases due to an increase of the number of bonds that determine the cluster resistance.

\section{Away from the percolation point}

When bonds with $\eta>\eta_{c}$ are added, the conductivity of the system increases. At sufficiently high values of $\eta$ the conductivity saturates and reaches a plateau value $\Sigma_{p}$. It is this conductivity that is of interest in many practical systems, because in reality one is not able to add or exclude bonds. According to CPA approaches, this plateau conductivity is closely related with the conductivity at percolation: ${ }^{7,9,10,31}$ $\Sigma_{p} \propto \beta e^{2} g_{0} \exp \left(-\eta^{\prime}\right)$ where $\eta^{\prime}$ is close to $\eta_{c}$.

For a variety of systems we have calculated the plateau conductivity. In Fig. 10 the logarithm of $\Sigma_{p} / \beta e^{2} g_{0}$ is presented as a function of $\left(\alpha^{2} \Delta B_{c}\right)^{1 / 3}$. From the data we calculated that $\eta^{\prime} /\left(\alpha^{2} \Delta B_{c}\right)^{1 / 3}=1.235 \pm 0.008$ (the dotted line), which is close to the obtained percolation threshold (see Sec. III A 4). This agreement is better than we reported in a recent paper, ${ }^{32}$ because in that study we did not divide out the prefactor of the conductivity $\beta e^{2} g_{0}$. Perhaps, the small difference between the percolation threshold and the slope of $\Sigma_{p} / \beta e^{2} g_{0}$ could be caused by the fact that other bonds then the critical one still slightly contribute to the network resistance.

\section{CONCLUSIONS}

We have studied VRH percolation in a 2D system. From our calculations we conclude that clusters generated with 


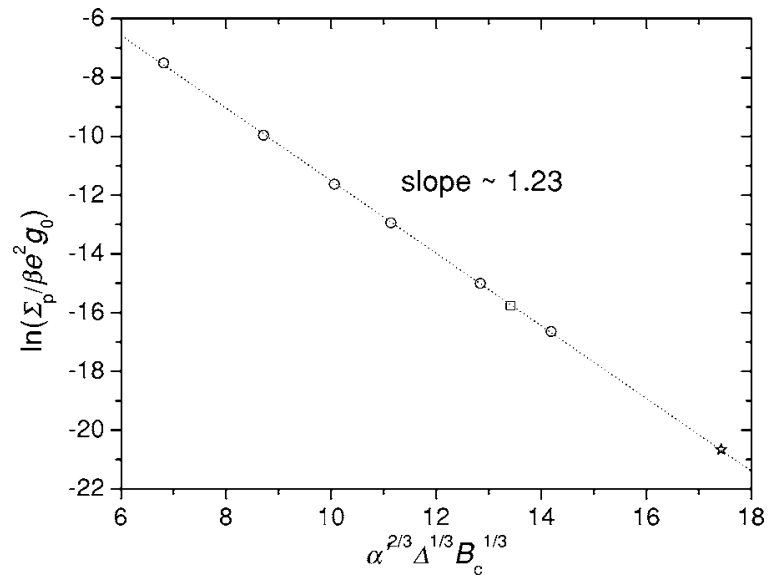

FIG. 10. The plateau conductivity for different systems: $\alpha=3$ $(\bigcirc), 4(\square)$, and 6 (污). Every datapoint is an average of 10-100 realizations.

VRH percolation are similar to the ones generated with ordinary lattice or continuum percolation algorithms. The obtained critical exponents and fractal dimensions indicate that VRH percolation belongs to the same universality class as the more standard percolation problems.

Further, we have shown that the concentration of sites participating in clusters $p$ varies linearly with $\eta$, which is a measure for the maximal resistance of the individual hops contributing to the conductivity of the system. As a consequence, the standard scaling laws for the behavior of the correlation length close to the percolation threshold are not changed by replacing $p$ by $\eta$.

A special feature of VRH is that the smallest length scale $r$, the distance between neighbours, depends on $\Delta$, the roughness of the energy landscape compared to $k_{B} T$, and the decay length of the interactions, $\alpha^{\prime-1}: r \propto\left(\Delta / \alpha^{\prime}\right)^{1 / 3}$. Via a similar reasoning as Mott followed in his derivation of the conductivity, ${ }^{6}$ it has been shown that this effective lattice constant is a consequence of the optimization of the length of a bond and the energy difference involved.

A subtle effect in VRH is the dependency of the critical coordination number $B_{c}$ on $r(\Delta$ and $\alpha)$. Especially at smaller values of $r$ the correlations between the sites seem to have more influence on the cluster morphology, leading to a decrease in this coordination number.

Our findings with respect to the universality class of VRH percolation, the scaling behavior of the effective lattice constant and the linear dependency of $\eta$ on the site concentration all indicate that the percolation arguments as used in CPA studies are applicable.

The conductivity calculations on just percolating clusters confirm the existence of the so-called critical subnetwork. An optimum in the cluster conductivity is found at a finite system size. Our findings disagree with CPA theories about the origin of the critical subnetwork in 2D. The optimum in the cluster conductivity originates from the fact that close the percolation threshold the number of bonds that influence the resistance increases.

The conductivity of networks away from the percolation point is still determined by the percolation threshold as predicted by CPA. Small deviations are observed, which are attributed to the contribution to the network resistance of other bonds then the critical ones.

\section{APPENDIX: PROOF OF EQ. (9)}

We consider a lattice of $M$ nodes. To each node $i$ a number $x_{i}$ is attributed that is randomly drawn from a uniform distribution with a width $W$ and bounds $\pm W / 2$. As a consequence there are no spatial correlations between the values $x$ of the nodes. The lattice is subdivided in $\tilde{M} \equiv M / m$ (each cell contains $m$ nodes). We characterize a cell by a number $\tilde{x}$, which is the value of $x$ that is closest to zero. We want to derive the probability distribution $P(\widetilde{x})$, which can be done as follows.

For every cell we have a set of $m$ numbers. We now divide the interval $[-W / 2, W / 2]$ into $n$ bins $(n \gg m)$. The bins are numbered from $-n / 2$ to $n / 2$. Note that the width of a bin is $W / n$. We will call a bin occupied when there exists a number with a value that is within the interval represented by that bin. The probability $\varphi$ that a bin is occupied equals $m / n$, given that $n \gg m$. We are interested in the probability $\Phi(k)$ that bin number $k$ is the occupied bin closest to the origin, which can be calculated with

$$
\Phi(k)=(1-\varphi)^{2|k|-1} \varphi .
$$

This equation can be rewritten as

$$
\Phi(k)=\varphi \exp (-2|k| \varphi),
$$

because $\varphi \ll 1(n \gg m)$.

The distributions $\Phi(k)$ and $P(\tilde{x})$ are related as follows:

$$
\Phi(k)=P(\tilde{x}) \Delta W / n,
$$

where $\tilde{x}=k W / n$. This relation holds as long as the variation of $P(\tilde{x})$ over the interval spanned by bin $k$ can be ignored. By combining Eqs. (A2) and (A3) we arrive at

$$
P(\widetilde{x})=\frac{m}{W} \exp (-2 m|\widetilde{x}| / W)
$$

which proves Eq. (9).

\footnotetext{
*Electronic address: h.p.huinink@tue.nl

${ }^{1}$ For a review, see, e.g., T. G. Castner, in Hopping Transport in Solids, edited by M. Pollak and B. I. Shlovskii (North-Holland, Amsterdam, 1991), p. 1.
}

${ }^{2}$ H. C. F. Martens, I. N. Hulea, I. Romijn, H. B. Brom, W. F. Pasveer, and M. A. J. Michels, Phys. Rev. B 67, 121203(R) (2003).

${ }^{3}$ J. A. Reedijk, H. C. F. Martens, H. B. Brom, and M. A. J. Mich- 
els, Phys. Rev. Lett. 83, 3904 (1999).

${ }^{4}$ Z. G. Yu and X. Song, Phys. Rev. Lett. 86, 6018 (2001).

${ }^{5}$ T. Vuletic, B. Korin-Hamzic, S. Tomic, B. Gorshunov, P. Haas, M. Dressel, J. Akimitsu, T. Sasaki, and T. Nagata, Phys. Rev. B 67, 184521 (2003).

${ }^{6}$ N. F. Mott, J. Non-Cryst. Solids 1, 1 (1968).

${ }^{7}$ V. Ambegaokar, B. I. Halperin, and J. S. Langer, Phys. Rev. B 4, 2612 (1971).

${ }^{8}$ M. Pollak, J. Non-Cryst. Solids 11, 1 (1972).

${ }^{9}$ M. Pollak, in The Metal-Non-Metal Transition in Disordered Systems, edited by L. R. Friedmand and D. P. Tunstall (University of Edinburgh, Edinburgh, 1978).

${ }^{10}$ B. I. Shklovskii and A. L. Efros, Electronic Properties of Doped Semiconductors (Springer-Verlag, Berlin, 1984), Chaps. 5 and 9.

${ }^{11} \mathrm{H}$. Böttger and V. V. Bryskin, Hopping Conduction in Solids (VCH Weinheim, Weinheim, 1985), Chaps. 4 and 5.

${ }^{12}$ A. S. Skal and B. I. Shklovskii, Sov. Phys. Solid State 16, 1190 (1974).

${ }^{13}$ G. E. Pike and C. H. Seager, Phys. Rev. B 10, 1421 (1974).

${ }^{14}$ C. H. Seager and G. E. Pike, Phys. Rev. B 10, 1435 (1974).

${ }^{15}$ J. A. McInnes and P. N. Butcher, Philos. Mag. B 39, 1 (1979).

${ }^{16}$ R. Lenormand and S. Bories, C. R. Seances Acad. Sci., Ser. B 291, 279 (1980).

${ }^{17}$ R. Chandler, J. Koplik, K. Lerman, and J. F. Willemsen, J. Fluid
Mech. 119, 24 (1982).

${ }^{18}$ D. Wilkinson and J. F. Willemsen, J. Phys. A 16, 3365 (1983).

${ }^{19}$ D. Wilkinson and M. Barsony, J. Phys. A 17, L129 (1984).

${ }^{20}$ M. M. Dias and D. Wilkinson, J. Phys. A 19, 3131 (1986).

${ }^{21}$ S. Schwarzer, S. Havlin, and A. Bunde, Phys. Rev. E 59, 3262 (1999).

${ }^{22}$ A. P. Sheppard, M. A. Knackstedt, W. V. Pinczewski, and M. Sahimi, J. Phys. A 32, L521 (1999).

${ }^{23}$ H. Cordes, S. D. Baranovskii, and J. Greif, Phys. Status Solidi B 230, 243 (2002).

${ }^{24}$ D. Stauffer and A. Aharony, Introduction to Percolation Theory, 2nd ed. (Taylor and Francis, London, 1994).

${ }^{25}$ M. Sahimi, Application of Percolation Theory (Taylor and Francis, London, 1994).

${ }^{26}$ C. M. Chaves and B. Koiller, Physica A 218, 217 (1995).

${ }^{27}$ E. T. Gawlinski and H. E. Stanley, J. Phys. A 14, L291 (1981).

${ }^{28}$ R. M. Ziff, Phys. Rev. Lett. 69, 2670 (1992).

${ }^{29}$ M. E. J. Newman and R. M. Ziff, Phys. Rev. E 64, 016706 (2001).

${ }^{30}$ P. H. L. Martins and J. A. Plascak, Phys. Rev. E 67, 046119 (2003).

${ }^{31}$ A. G. Hunt, Adv. Water Resour. 279, 24 (2001).

${ }^{32}$ W. F. Pasveer, P. A. Bobbert, H. P. Huinink, and M. A. J. Michels, Phys. Rev. B 72, 174204 (2005). 